\title{
Application of Path Duration Study in MultiHop Ad Hoc Networks
}

\author{
Alicia Triviño-Cabrera, Jorge García-de-la-Nava, Eduardo Casilari, and Francisco \\ J. González-Cañete
}

Dpto. Tecnología Electrónica, Universidad de Málaga, Campus Teatinos, 29071 Málaga, Spain

\{atc, ecasilari,fgc\}@uma.es

\begin{abstract}
Some ad hoc protocols allow the discovery and/or storage of multiple routes to the same destination node. The selection of the path to utilize is commonly based on the criterion of the minimum number of hops. However, other strategies could be more appropriate to improve the network performance. In this sense, this paper proposes the criterion based on the estimation of the Mean Residual Path Lifetime. In order to compute this metric, a formal description of link duration in mobile ad hoc networks is presented. The model has been verified by means of simulations enclosing different mobility and transmission conditions. From the link duration model, an analytical expression for path duration and mean residual lifetime is then derived. Finally, the authors show that selected ad hoc routing paths live longer when the proposed criterion is employed.
\end{abstract}

Keywords: MANET, Path Duration, Route Selection.

\section{Introduction}

Mobile Ad Hoc NETworks (MANET) are formed by wireless devices that intercommunicate without the utilization of any centralized entity that manages the radio resources. As direct communication is only possible for neighbor nodes, the transmission of information between distant devices is supported by the cooperation of intermediate nodes that retransmit and route their packets. The sequence of intermediate nodes involved in the retransmission process constitutes a route or path. Due to the mobility of the nodes that are part of the established paths, routes present a finite lifetime (path duration). When the utilized route is down, the source node should initiate the procedure to discover a new path in order to continue the on-going communications. As this procedure is usually associated to a controlled packet flooding, the route discovery implies an increment in the network overhead and communication delay. Taking into account the energy consumption of the retransmissions as well as the wireless interferences associated to the flooding that may cause packet losses, the employ of route discoveries techniques should be minimized. In this sense, the criterion for selecting paths plays an important role. For 
two communicating nodes, there may exist multiple paths among which one route must be selected for the communication. Although the criterion of the minimum number of hops is commonly utilized, diverse metrics could be employed for the selection. Specifically, the consideration of the expected path duration could be advantageous so longer to live paths would be preferred to routes associated to shorter path durations.

Despite of the potential benefits that could be achieved if the employ of the expected path duration is considered, the a-priori computation of this parameter is not an easy issue as it requires the pre-determination of the future node movements. In order to overcome this limitation, other available information is utilized for the estimation of the residual path duration. For instance, some authors proposed that there is a direct relationship between the signal strength of the received packets along a path and its residual lifetime [2]. However, different propagation conditions may provoke that the previous assumption does not hold. From another point of view, the authors in [3] show that the inclusion of the mean link duration in the selection criterion implies the utilization of paths that live longer. This work is supported by approximating link and path duration to an exponential function, i.e. a memory-less model where the time that the route has been active since its creation is not considered. Although this approximation may become valid for those paths with a high number of hops [4], this condition is not satisfied in conventional ad hoc applications where paths usually contain 2 or 3 hops [5]. Under these circumstances, the present work proposes the lognormal fitting for the link duration in heterogeneous scenarios. From this model, the mean residual lifetime is computed. As the results show that the expected mean residual lifetime does depend on the survival time, this information is employed for the estimation of the path residual lifetime in the proposed criterion. The simulations show that the proposed criterion is associated to the selection of paths with greater duration.

The rest of the paper is structured as follows. Section 2 presents the related work. Section 3 shows the results of analyzing the link duration in heterogeneous scenarios. This analysis is utilized for the computation of the mean residual path lifetime presented in Section 4. With this parameter, a criterion for selecting paths is constructed as described in Section 5. The benefits associated to the employ of this criterion are checked by means of extensive simulations whose results are shown in Section 6. Finally, Section 7 draws the main conclusion of this work.

\section{Related Work}

In order to compute the mean residual path lifetime, that is, the time that an already established path is expected to live, a characterization of path duration is required.

Simulation has been the main method for analyzing the properties of path duration in ad hoc networks. One of the first studies concerning the analysis of path duration was due to Bai et al. [6]. Basing on experimental results obtained by simulations, these authors assume that the lifetime of a path with four or more hops can be approximated to an exponential distribution. However the study does not consider the fit of any other standard distribution. Moreover, the selection of an exponential 
distribution is not justified with any mathematical validation. To cope with this lack, Han et al., basing their work on Palm's theorem, state that, under some circumstances, the lifetime associated to those paths with a large number of hops converges to an exponential distribution [4]. The exponential fitting has also been utilized in [7] [8].

Another remarkable work related to the exponential fitting is described in [3]. In this study, each path is associated to a parameter $\left(\lambda_{P A T H}\right)$ of the exponential fitting of the route duration. The $\lambda_{P A T H}$ of the route is derived from the $\lambda$ of the links $\left(\lambda_{L I N K}\right)$ that compose the route. With this purpose, the nodes estimate the $\lambda_{L I N K}$ as the inverse of the mean link duration in an interval of time (auto-regresive filters are suggested for this estimation). The path to employ is the one with a lowest $\lambda_{P A T H}$, which is assumed to be related to longer path duration.

In spite of the popularity of exponential fitting for link duration in MANETs, this approximation presents a common disadvantage as they provide a solution for the analysis of paths which is valid only for routes with a large number of hops. Therefore, their study could not be fully applied to usual ad hoc networks and practical MANET applications where most paths normally only consist of 1 to 4 hops [5]. The importance of short paths is reinforced by the fact that the most relevant protocols utilize the minimum hop count as the metric to select the route in use in order to reduce the effects of the wireless medium on the performance of the network.

\section{Link Duration}

Although link duration is considered a fundamental parameter when evaluating the mobility in a MANET [9], few studies provide a formal description of this variable. Some authors have formally studied the link duration for specific mobility models. For instance, [10] have studied the mean value of link duration under a constant velocity model meanwhile [11] analyzed it for a deterministic, partially deterministic and Brownian mobility models. On the other hand, [12] formally describes the statistics associated to the link duration for a simplified random mobility model. In [13], a thorough analytical study of some significant parameters of link duration is presented. The main disadvantage is that it is only valid for the Constant Velocity Model.

In this section, we approach the fitting of the link duration in heterogonous scenarios characterized by different mobility models. The following standard distributions are considered: Normal, Gamma, Weibull, Rayleigh, Pareto, Exponential and Lognormal. The parametrization of the previous approximations are based on the correspondence of the two first moments of the data to the considered function moments. Similar results have been obtained if Maximum Likehood Estimators (MLE) are employed. Then, we evaluate the fittings by means of the KolmogorovSmirnof goodness-of-fit test (K-S test). The K-S test is an extended tool that captures the maximum deviation of the hypothesized Cumulative Distribution Function respect to the data to approximate.

The computation of link duration is obtained by the utilization of a developed module that was incorporated into the Matlab tool [14]. The basis of this module resides in the computation of the connectivity graph. A connectivity graph informs 
about the nodes that are neighbors, i.e., nodes that are directly connected because the distance that separates them is lower than the transmission range. Differences in the evolution of the connectivity graph imply the break or the creation of links.

The status of a wireless link depends on numerous system and environmental factors that impact both the transmitter and the receiver ranges. Although the transmission range of a node experiments spatial and temporal variations that concludes in a coverage area that is neither fixed nor symmetric, the exclusion of the modeling of these conditions simplifies the analysis of the network performance. Therefore the authors will consider the transmission range as a perfect circumference (in a two-dimensioned scenario).

In order to analyze link duration in the wide variety of scenarios where MANETs are expected to be deployed, two opposite mobility patterns are employed in order to conclude that the lognormal fitting is the best. Firstly, the Random WayPoint is utilized as it is quite extended in the studies of ad hoc network evaluation. We have varied the main parameters of this synthetic model. On the other hand, real movement traces are employed.

\subsection{Random WayPoint}

One of the most extended individual mobility models is the Random WayPoint (RWP). According to this pattern, the nodes of an ad hoc network move along a straight line between two destination points (waypoints) placed in a finite space. In the literature, this space is normally bi-dimensional and restricted to a rectangular area of dimensions $x_{\max }$ and $y_{\max }$. Once a node reaches a destination point, a new one is uniformly selected from this area. The speed for a movement is also chosen from a uniform distribution in the interval $\left[\mathrm{v}_{\min }, \mathrm{v}_{\max }\right]$. Both speeds and waypoints are generated independently of all the previous destinations and speeds. In addition, the model allows nodes to pause between two consecutive trips for a certain period of time. This period (Pause Time) is habitually fixed to a constant value. By varying the values of the transmission range of the nodes, $x_{\max }, y_{\max }, v_{\min }, v_{\max }$ and the pause time, it is possible to control the movement conditions of the simulated scenario.

The generic formulation of the Random WayPoint was proved to be unreliable by [15]. In order to suppress the instability in the results, authors in [15] propose the incorporation of a simple modification that consists of fixing a minimum not null speed in the nodes. This mobility pattern is called the Modified Random WayPoint. Due to its popularity as well as its capability of characterize multiple scenarios, the authors of this contribution will continue the analysis of path duration assuming nodes follow the Modified Random WayPoint Model.

Despite the Random WayPoint is widely utilized as a mobility pattern, at present, no analytical formulation has related the statistical properties of the link duration to the parameters of the modified RWP, the network dimensions, the node density or the transmission range. The references dealing with this issue are all based on simulations [16]. In general, the authors suggest the approximation to a standard distribution function without any mathematical support [6] [17].

In order to simulate the conditions on which multi-hop wireless networks can be deployed, we have evaluated the link duration in a wide set of experiments. The 
experiments estimates the link duration under different values of the area shape $\left(1500 \times 300 \mathrm{~m}^{2}, 1000 \times 1000 \mathrm{~m}^{2}\right)$, the maximum speed $(5,10,15 \mathrm{~m} / \mathrm{s})$, the pause time $(0$, $50 \mathrm{~s})$, the node density $(15,50$ nodes $)$ and the transmission range $(250,100 \mathrm{~m})$ in 10000 -second simulations. Additionally, the distribution of link duration is also computed from traces obtained when the NS-2 Simulator tool is employed [18]. Because of space limitations, the results of the K-S tests are averaged and exposed in Figure 1 where the straight lines represent the standard deviation of the test statistics. As it can be observed, the best approximation is clearly obtained by a lognormal distribution function. It must be noted that for each of the performed simulations, the lognormal distribution is always the best fit according to the K-S test.

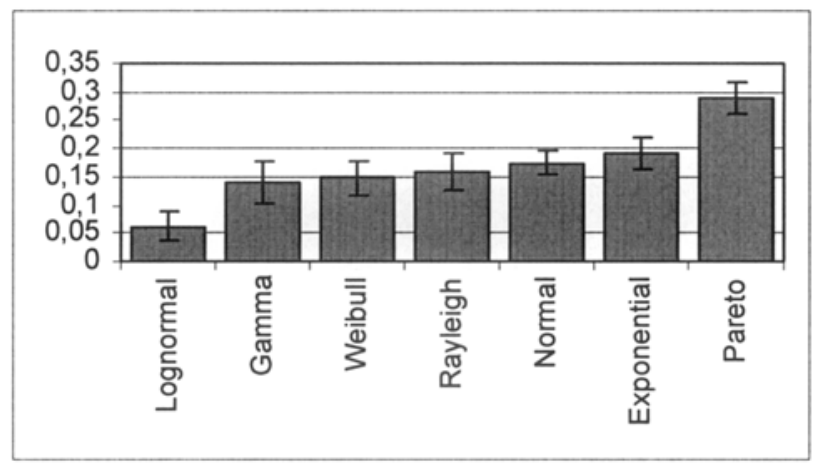

Fig. 1. Mean and deviation results of the K-S tests over 50 scenarios.

\subsection{Real Mobility Traces}

In order to study link duration in a VANET (Vehicular Ad Hoc Network) scenario, real position traces extracted from the public buses in Seattle are employed to compute link duration [19]. These traces are collected by the periodic transmission of the buses informing about their position along two days in 2001. Straight trajectories are assumed between consecutive position data. As conventional DSRC (Direct Short Range Communication), the transmission range was set to $1500 \mathrm{~m}$. With these data, the connectivity graph is updated each second.

The fitting of the link duration is accomplished by means of the K-S test. The Fig. 2 shows that the best approximation corresponds to the lognormal function again. 


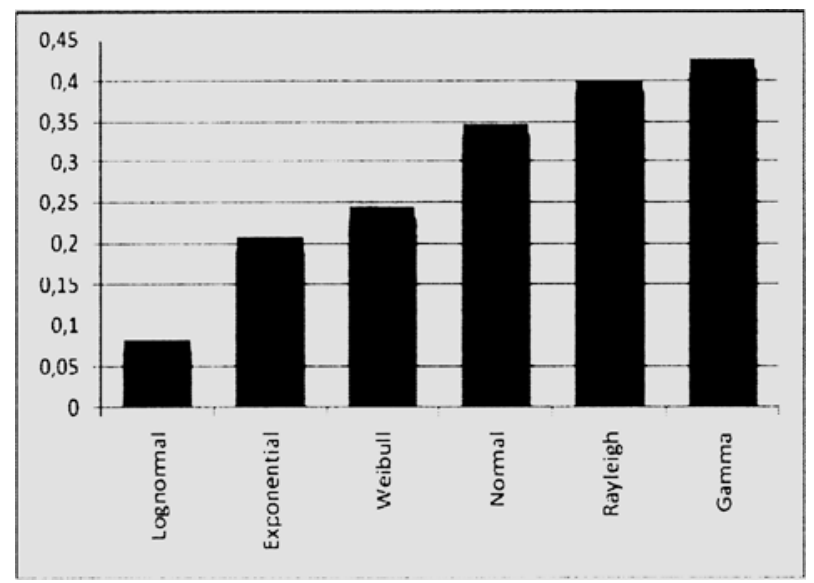

Fig. 2. Results of the K-S test for Link Duration Fitting in a VANET scenario.

\subsection{Lognormal Fitting}

From the previous sections, we can conclude that link duration could be accurately approximated by a lognormal function. This function is usually employed in the reliability research area as it is able to model the lifetime of electronic devices.

Formally, the probability that a link lifetime $(L)$ is lower than $t(F(t))$ is computed by the Eq. 1 where $\mu \mathrm{y} \sigma$ are the parameters of the lognormal function. These parameters are correlated to the characteristics of the scenario.

$$
F(t)=\frac{1}{2}+\frac{1}{2} \operatorname{erf}\left(\frac{\log (t / \mu)}{\sigma \sqrt{2}}\right)
$$

From this model, the path duration could be analytically described. The results are presented in [20].

\section{Mean Residual Path Lifetime}

In reliability theory, the mean residual life (MRL) is often used as a metric of the time that a component is expected to work given that the component has already lived a survival time $t$. In wireless multi-hop networks, this parameter can be employed as a measure of the stability associated to the routes of the nodes.

Formally, the MRL is defined as [21]:

$$
\operatorname{MRL}(t)=E[X-t / X>t]=\frac{\int_{t}^{\infty} \bar{F}(u) d u}{\bar{F}(t)}=\frac{\int_{t}^{\infty}\left(\frac{1}{2}-\frac{1}{2} \operatorname{erf}\left(\frac{\log (u / \mu)}{\sigma \sqrt{2}}\right)\right) d u}{\frac{1}{2}-\frac{1}{2} \operatorname{erf}\left(\frac{\log (t / \mu)}{\sigma \sqrt{2}}\right)}
$$


where $\vec{F}(t)=1-F(t)$ corresponds to the survavility function, i.e., the Complementary Cumulative Distribution Function or CCDF. In this case, the $F(t)$ corresponds to the distribution function of path duration which is analytically derived from link duration model in [20].

Figure 3 plots the MRL curves for different number of hops when all the links are characterized by the same $\mu$ and $\sigma$. It is shown that MRL values are strongly dependent on the number of hops. Additionally, they also vary on the survival time in a non-monotonic way as it corresponds to a lognormal-like MRL. The figure shows that this variation is smoothed when the number of hops increases. The limit when the number of hops is high makes the MRL curve tend to a constant value. Constant MRLs are related to exponential PDFs which is coherent with Palm's Theorem [4].

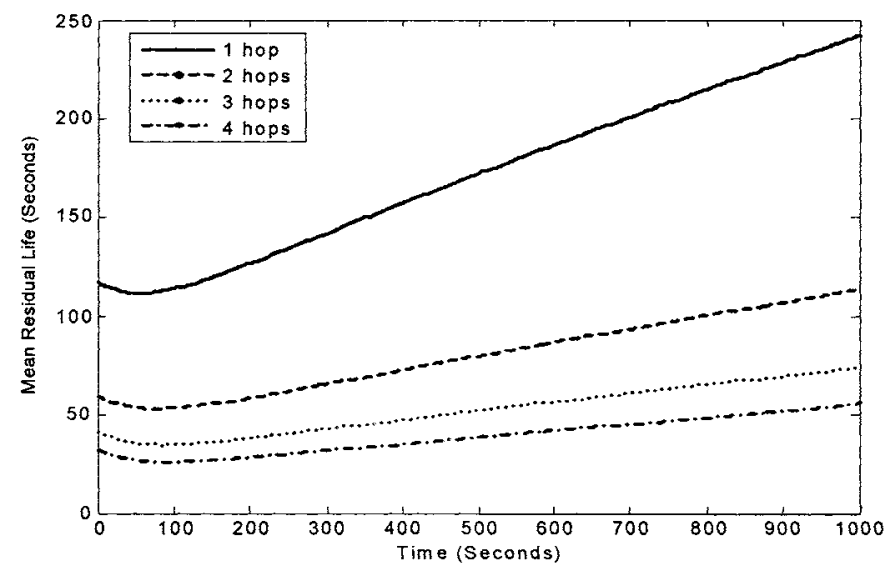

Fig. 3. MRL plots for $1,2,3$ and 4 hops. Scenario parameters are: Uniform speed, $V_{\max }=2$ $\mathrm{m} / \mathrm{s}, \mathrm{V}_{\min }=1 \mathrm{~m} / \mathrm{s}$, Pause $=0 \mathrm{~s}$, Tx range $=250 \mathrm{~m}$, Nodes $=50$, Area $=1500 \times 300 \mathrm{~m}^{2}$.

\section{Proposed Criterion for Selecting Paths}

Some ad hoc routing protocols allow the discovery of multiple routes to the same destination node. Under these circumstances, a criterion for selecting the route to employ is required. In this paper, a criterion based on the estimation of the path MRL is proposed. Specifically, among the paths with minimum number of hops, the route with a greater estimated MRL is selected. We consider that paths with minimum number of hops are preferred so that the number of wireless links can be minimized. The utilization along longer paths may increase the interferences as packets are retransmitted in each of the links that are part of the route.

To compute the Path MRL employing Eq. 2, the node that selects the path requires the parameters $\mu_{i}$ and $\sigma_{i}$ of each $i$ link within the path as well as the time when the link was established for a $N$-hop path $(1 \leq i \leq N)$. Therefore, $3 \cdot N$ extra fields should be 
included in the ad hoc routing packets (Route Request or Route Reply). This is a clear limitation as it could noticeably increase the header length of packets. In order to overcome this restriction, each node estimates the MRL of the link that is going to be employed for the retransmission of the routing packet. From these data, the path MRL is approximated in a similar way to [3]. Formally, Eq. 3 shows the estimation of the Path MRL in a $N$-hop path:

$\frac{1}{M R L_{-} P A T H}=\sum_{i=1}^{N} \frac{1}{M R L_{-} \text {Link }_{i}}$

Therefore, only one extra field (MRL_field) is incorporated into the header of the routing packets. This field accumulates the inverse of the MRLs of the links in the paths. As routes are learnt by the reception of Route Request (RREQ) and Route Reply (RREP) messages, the format of both packets should be modified. The Fig. 4 illustrates the process of retransmitting RREP packets and how the MRL field is updated. In the example of the Figure 4, to select a route to Node C, Node A will select the path associated to a minimum value in the MRL_field as this field stores the inverse of the estimated path MRL.

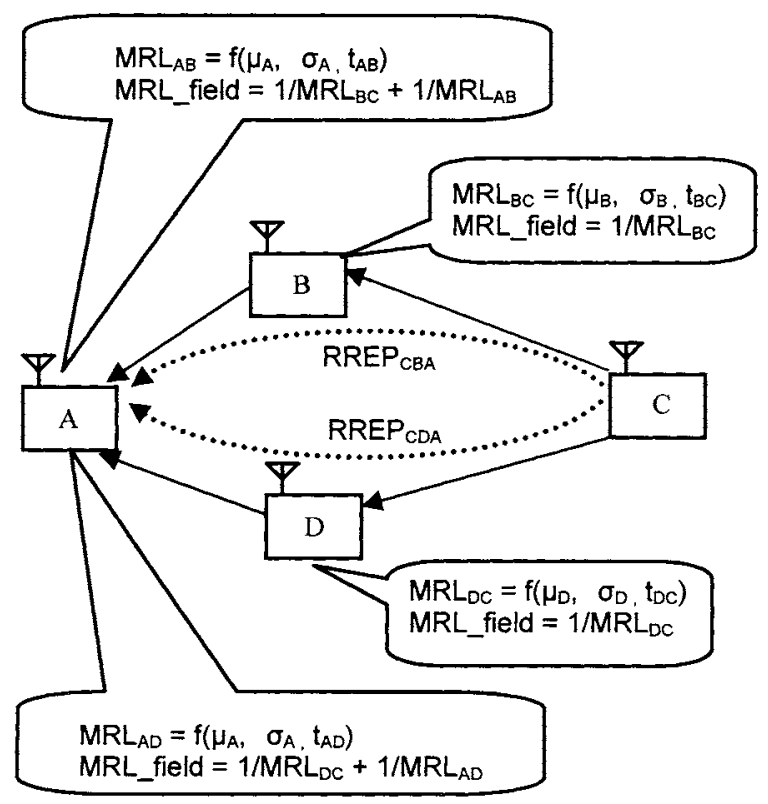

Fig. 4. Scheme for the retransmission of routing packets where the MRL_field is updated.

To compute the Link MRL, the $i$-th node utilizes the parameters $\mu_{i}, \sigma_{i}$ and the time when the link with the previous node $j$ was established $t_{i j}$. The latter parameter could be easily stored in the neighbor list [22]. However, the $\mu_{i}$ and $\sigma_{i}$ could only be 
estimated through the monitoring of the links. In [3], the $\lambda$ parameter is derived from the observed mean link duration by means of an auto-regressive filter. In an analogous way, we estimate the $\mu_{i}$ and $\sigma_{i}$ from the lifetimes associated to the links that are down in a given interval of time $\left(T_{E W M A}\right)$. We compare this technique to the estimation of $\mu_{i}$ and $\sigma_{i}$ from the lifetimes of the links that are at that moment up. The results show that there exist little differences when employing them. Therefore, we utilized the second method as its implementation is simpler.

\section{Simulation Results}

To evaluate the proposed criterion, a new module was developed in a Matlab framework [14]. In each 100000-second simulation, a source and a destination are randomly chosen. Then, four paths are selected accordingly to the different analyzed criteria. When one of the paths is broken, the path duration is stored in the data associated to the criterion by which the path was selected. The decision process is repeated once the four paths are down selecting again another set of four paths.

In each decision point, among the paths with a minimum number of hops, four of them are selected according to the following criteria:

- Maximum MRL Path. The path with the greatest estimation of the MRL is selected.

- Minimum $\lambda$ Path. The path with a lowest $\lambda$ is chosen. This criterion is included in order to compare the proposed criterion to the metric described in [3].

- Minimum MRL Path. The path with the lowest estimated MRL is selected. This criterion is included in order to establish a limit in the network performance.

- First Path. The first discovered path is employed. The route is randomly chosen among all the candidates with the minimum number of hops. This criterion does not consider any MRL estimation.

Five different scenarios characterized by a constant speed are employed for the evaluation. The simulation parameters are summarized in Table 1. The Fig. 5 draws the simulation results. As it is shown, the application of the Maximum MRL Path Criterion implies the selection of paths with longer lifetimes. These lifetimes exceed those obtained when the Minimum $\lambda$ Path Criterion is employed.

Table 1. Parameters of the simulations.

\begin{tabular}{|l|l|}
\hline Simulation Area & $1500 \mathrm{~m} \times 300 \mathrm{~m}$ \\
\hline Mobile nodes & 50 \\
\hline Mobility pattern & $\begin{array}{l}\text { Constant Speed: }[1,5] \mathrm{m} / \mathrm{s} . \\
\text { Pause Time : } 0 \text { seconds }\end{array}$ \\
\hline Simulation Time & $100000 \mathrm{~s}$ \\
\hline Transmission Range & $250 \mathrm{~m}$ \\
\hline
\end{tabular}




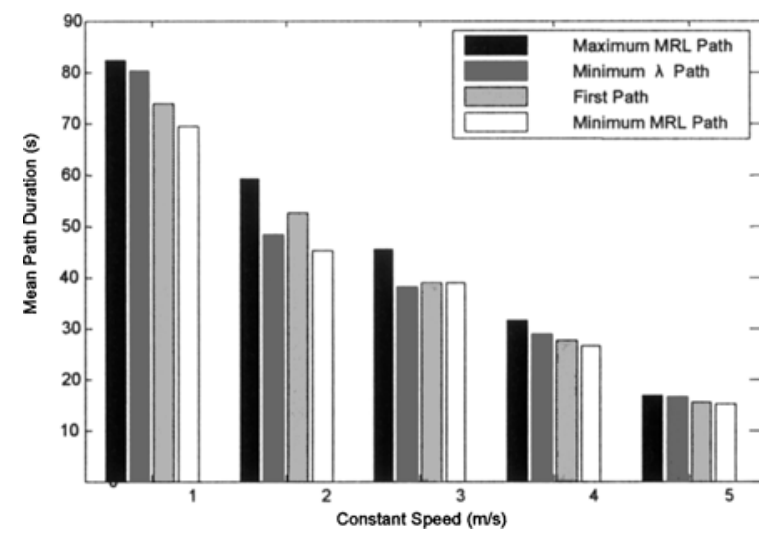

Fig. 5. Mean Path Duration associated to the application of 4 Path selection Criteria.

\section{Conclusions}

This paper presents two significant contributions. Firstly, the authors show that the link duration can be accurately approximated by a lognormal function in heterogeneous scenarios. Secondly and based on the previous result, the authors analytically compute path duration and path mean residual lifetime. From this study, it is possible to show that a memory-less model could only be employed when the number of hops that the route contains is high (greater than 4). For routes involved in conventional ad hoc applications (with 2 o 3 hops), the criterion for selecting routes in the network could be supported by the path survival time, i.e., the time elapsed since the route was established. The path mean residual lifetime is estimated by means of the link residual lifetime associated to the links that form the path. This data is included in the criterion for selecting routes in multihop ad hoc networks. The simulation results show that path live longer when this information is incorporated in the decision phase of path selection.

Acknowledgments. This work has been partially supported by the Public Spanish Project TEC2006-12211-C02-01.

\section{References}

1. S. Gwalani, E. M. Belding-Royer and C. E. Perkins, "AODV-PA: AODV with path accumulation," Communications, 2003.ICC'03.IEEE International Conference on, vol. 1, 2003. 
2. O. Tickoo, S. Raghunath and S. Kalyanaraman, "Route fragility: a novel metric for route selection in mobile ad hoc networks," Networks, 2003.ICON2003.the 11th IEEE International Conference on, pp. 537-542,

3. Y. Han and R. J. La, "Maximizing Path Durations in Mobile Ad-Hoc Networks," 40th Annual Conference on Information Sciences and Systems, Princeton, NJ, March, 2006.

4. Y. Han, R. J. La and A. M. Makowski, "Distribution of path durations in mobile ad-hoc networks--Palm's theorem at work," 16th ITC Specialist Seminar, 2004.

5. J. Broch, D. A. Maltz, D. B. Johnson, Y. C. Hu and J. Jetcheva, "A performance comparison of multi-hop wireless ad hoc network routing protocols," Proceedings of the 4th Annual ACM/IEEE International Conference on Mobile Computing and Networking, pp. 85-97, 1998.

6. F. Bai, N. Sadagopan, B. Krishnamachari and A. Helmy, "Modeling path duration distributions in MANETs and their impact on reactive routing protocols," Selected Areas in Communications, IEEE Journal on, vol. 22, pp. 1357-1373, 2004.

7. S. Arbindi, K. Namuduri and R. Pendse, "Statistical estimation of route expiry times in ondemand ad hoc routing protocols," Mobile Adhoc and Sensor Systems Conference, 2005.IEEE International Conference on, pp. 16-23, 2005.

8. S. Jiang, D. He and J. Rao, "A prediction-based link availability estimation for mobile ad hocnetworks," INFOCOM 2001.Twentieth Annual Joint Conference of the IEEE Computer and Communications Societies.Proceedings.IEEE, vol. 3, 2001.

9. J. Boleng, W. Navidi and T. Camp, "Metrics to enable adaptive protocols for mobile ad hoc networks," Proceedings of the International Conference on Wireless Networks (ICWN'02), pp. $293298,2002$.

10. S. Cho and J. P. Hayes, "Impact of Mobility on Connection Stability in Ad Hoc Networks," Proc. of IEEE Communication Society, WCNC, vol. 3, pp. 1650-1656, 2005.

11. D. Turgut, S. K. Das and M. Chatterjee, "Longevity of routes in mobile ad hoc networks," Vehicular Technology Conference, 2001.VTC 2001 Spring.IEEE VTS 53rd, vol. 4, 2001.

12. A. B. McDonald and T. Znati, "A path availability model for wireless ad-hoc networks," Wireless Communications and Networking Conference, 1999.WCNC.1999 IEEE, pp. 35-40, 1999.

13. P. Samar and S. B. Wicker, "On the behavior of communication links of a node in a multihop mobile environment," Proceedings of the 5th ACM International Symposium on Mobile Ad Hoc Networking and Computing, pp. 145-156, 2004.

14. http://www.mathworks.com

15. J. Yoon, M. Liu and B. Noble, "Random waypoint considered harmful," INFOCOM 2003.Twenty-Second Annual Joint Conference of the IEEE Computer and Communications Societies.IEEE, vol. 2 ,

16. M. Gerharz, C. de Waal, P. Martini and P. James, "Strategies for finding stable paths in mobile wireless ad hoc networks," Local Computer Networks, 2003.LCN'03.Proceedings.28th Annual IEEE International Conference on, pp. 130-139, 2003.

17. T. Dimitar, F. Sonja, C. Bekim and G. Aksenti, "Link Realiability analysis in ad hoc networks," Proc. of XII Telekomunikacioni Forum TELFOR, 2004.

18. K. Fall and K. Varadhan, "The ns Manual (formerly ns Notes and Documentation)," The VINT Project, vol. 1, 2002.

19. J. G. Jetcheva, Y. C. Hu, S. PalChaudhuri, A. K. Saha and D. B. Johnson, "Design and evaluation of a metropolitan area multitier wireless ad hoc network architecture," Mobile Computing Systems and Applications, 2003. Proceedings.Fifth IEEE Workshop on, pp. 32-43, 2003.

20. A. Triviño-Cabrera, J. Garcia-de-la-Nava, E. Casilari and F. J. González-Cañete, "An analytical model to estimate path duration in MANETs," Proceedings of the 9th ACM International Symposium on Modeling Analysis and Simulation of Wireless and Mobile Systems, pp. 183-186, 2006. 
74 PWC 2007

21. N. Ebrahimi, "Estimation of Two Ordered Mean Residual Lifetime Functions", Biometrics, Vol. 49, No. 2 (Jun., 1993), pp. 409-417

22. C. E. Perkins, E. Belding-Royer and S. Das, "Ad hoc on demand distance vector (AODV) routing. IETF RFC 3561, 2003. 